Y. C. Minh and E. F. van Dishoeck, eds.

\title{
The Atmospheres of Substellar-Mass Objects
}

\author{
Adam Burrows \\ The University of Arizona, Department of Astronomy, 933 N. Cherry \\ Ave., Tucson, AZ 85721, USA
}

\begin{abstract}
Between the masses of the Jovian planets and the stars, a new population of exotic substellar-mass objects (SMOs) is now being discovered in profusion, that are challenging our notions of what a planet, brown dwarf, or star can be. I focus on some exotic and distinctive aspects of the compositions of SMO atmospheres. In particular, I review the formation of silicate, water, and ammonia clouds, the role of rainout in altering the abundance of the alkali metals, and the suppression by atomic potassium of the optical fluxes of a methane dwarf such as Gliese 229B. Furthermore, I discuss the order in which various molecular, atomic, and condensed species might dominate or influence SMO atmospheres, with an eye to anchoring new spectral classes from $T_{\text {eff }} \sim 2000 \mathrm{~K}$ to $\sim 100 \mathrm{~K}$.
\end{abstract}

\section{The Chemical Reservoirs of the Elements in SMO Atmospheres}

The molecular compositions of extrasolar giant planet (EGP) and brown dwarf atmospheres can serve as diagnostics of temperature, mass, and elemental abundance and can help define a spectral sequence, just as the presence or absence of spectral features associated with various ionization states of dominant, or spectroscopically active, atoms and simple molecules does for $\mathrm{M}$ through $\mathrm{O}$ stars. Figure 1 depicts some of the chemical reservoirs (in "balloons") of the elements, themselves indicated on the figure at their position in atomic number/abundance space (Anders \& Grevesse 1989). However, the multiplicity of molecules that appear in their atmospheres lends an additional complexity to the study of substellar mass objects (SMO) that is helpfully diagnostic. Nowhere is the latter more apparent than in the appearance at low temperatures of refractory grains and clouds. These condensed species can contribute significant opacity and can alter an atmosphere's temperature/pressure profile and its albedo. Grain and cloud droplet opacities depend upon the particle size and shape distribution and these are intertwined with the meteorology (convection) in complex ways. Furthermore, condensed species can rain out and deplete the upper atmosphere of heavy elements, thereby changing the composition and the observed spectrum. As demonstrated in the Gl 229B campaign and in attempts to fit its spectrum, there is strong evidence that heavy metals, such as $\mathrm{Ca}, \mathrm{Fe}, \mathrm{Ti}, \mathrm{V}, \mathrm{Si}, \mathrm{Al}$, and $\mathrm{Mg}$, are indeed depleted in Gl 229B's atmosphere (Nakajima et al. 1995; Oppenheimer et al. 1995, 1998; Marley et al. 1996; Allard et al. 1996). In addition, such depletions are manifest in the atmospheres of Jupiter and Saturn. This is altogether to be expected, since the grains that condense below temperatures of 


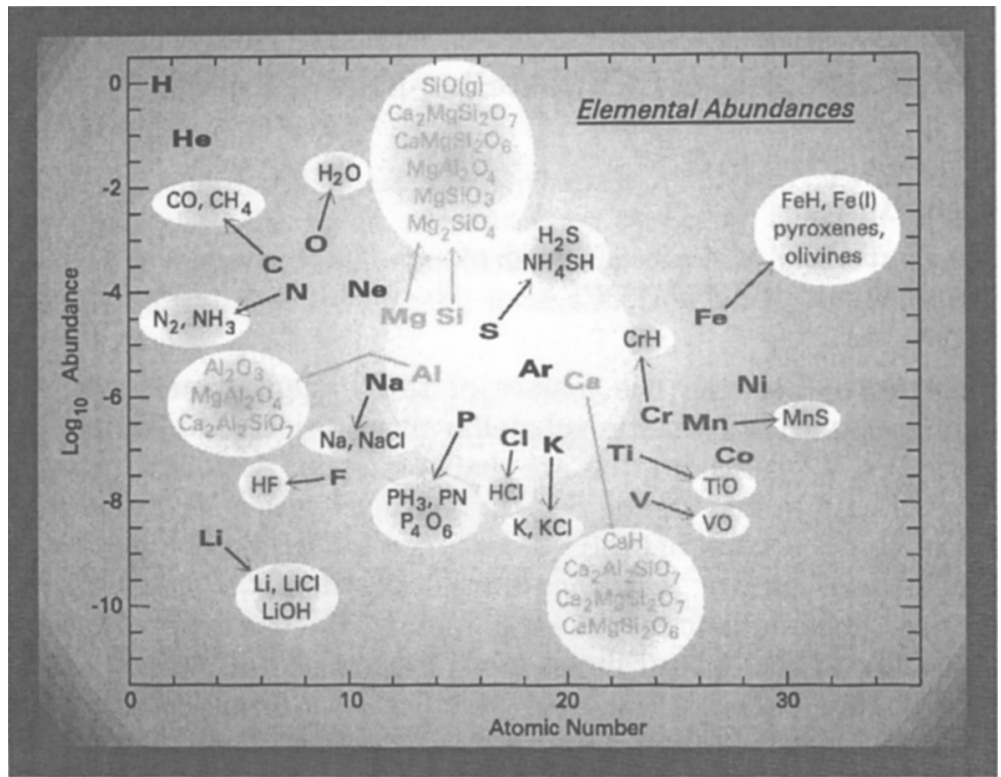

Figure 1. The $\log _{10}$ of the abundances of the major elements versus atomic number. The arrows guide the eye to "balloons" that contain a collection of some of the major compounds in which these elements reside in EGP and brown dwarf atmospheres.

$\sim 2500 \mathrm{~K}$ should form droplets and rain out of the atmosphere. This "rainout" will carry the condensate's elements to depth. The lower boundary of the grain cloud deck should be near where the object's temperature-pressure profile intersects the condensate's equilibrium lines, but its upper boundary is more difficult to determine.

The physical extent of the clouds is a function of the character of convection and the meteorology of the atmosphere. The droplet sizes are determined by the poorly-understood processes of nucleation, coagulation, and breakup. Furthermore, the high opacity of the grains can turn an otherwise radiative zone into a convective zone, and, thereby, influence the cloud extent and composition. In principle, if a convective zone were well-mixed and the chemical kinetics were suitably fast, the compositions in the convective zones would closely follow the equilibrium abundance patterns. However, the condensibles are likely to settle and concentrate at depth. In short, due to the low temperatures of substellar atmospheres, we would expect a variety of cloud layers with a variety of compositions both above and below their photospheres. Which clouds dominate is expected to be a function of the object's effective temperature, gravity, and metallicity. In brown dwarf and EGP atmospheres, abundance and temperature/pressure profiles, particle properties, spectra, and meteorology are inextricably linked. 


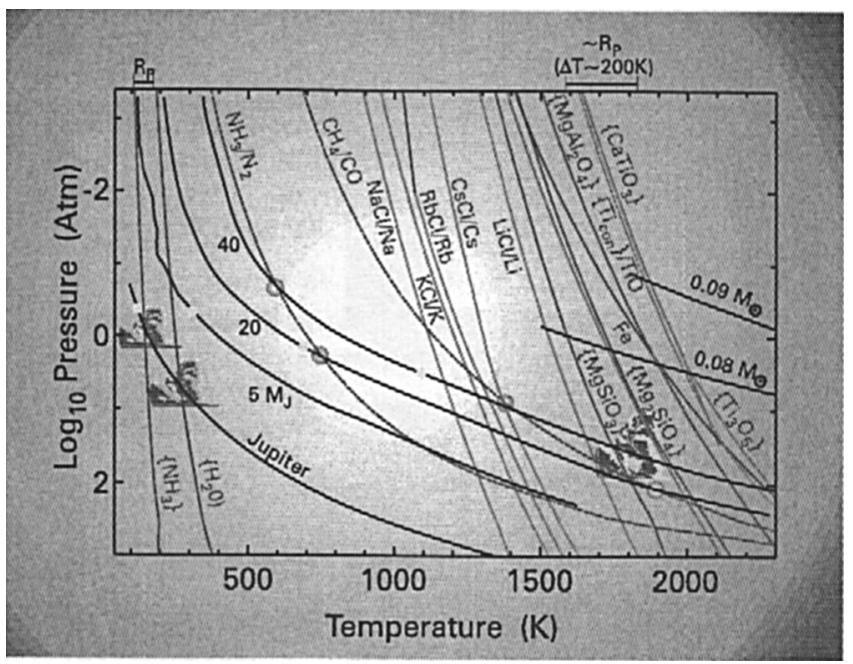

Figure 2. Taken from the work of Burrows \& Sharp (1999), this figure depicts various temperature-pressure profiles of SMOs at a 1 Gyr age, along with equilibrium lines for various compositional transitions such as $\mathrm{CH}_{4} / \mathrm{CO}, \mathrm{NH}_{3} / \mathrm{N}_{2}$, and $\mathrm{K} / \mathrm{KCl}$. Also shown are condensation lines for some relevant refractories. The clouds indicate the approximate positions for the bases of the $\mathrm{NH}_{3}, \mathrm{H}_{2} \mathrm{O}$, and silicate clouds.

Figure 2 depicts the regions of transition from one chemical species to another in temperature-pressure space for a variety of important constituents of equilibrium substellar atmospheres, taken from the work of Burrows \& Sharp (1999). Also shown are the regions of $T / P$ space in which we would expect to find $\mathrm{NH}_{3}, \mathrm{H}_{2} \mathrm{O}$, and silicate clouds. Only a subset of the calculated and interesting composition boundaries are depicted. Superimposed on this plot is an atmospheric profile of Jupiter, as well as 5, 20, and $40 \mathrm{MJ}_{\mathrm{J}}$ models at $1 \mathrm{Gyr}$. Also shown for comparison on Figure 2 are adiabats below the photospheres for more massive $\mathrm{M}$ dwarf models of Burrows et al. (1993) at 0.08 and $0.09 \mathrm{M}_{\odot}$ and 1 Gyr. The near verticality of the transition curves confirms that composition is most closely linked with temperature. Figure 2 summarizes some of the diagnostic chemical sequences and transitions encountered in theoretical substellar atmospheres and the reservoirs of the major elements (see also Figure 1).

Inspired by diagrams like Figure 2, we can put together a hierarchy of appearances and diappearances that can help to define the spectroscopic subclasses of the L dwarfs (Kirkpatrick et al. 1999), the methane dwarfs, and lowertemperature $\left(T_{\text {eff }} \leq 700 \mathrm{~K}\right)$ classes yet to be discovered, all the way down to the Jovian planets of our solar system $\left(T_{\text {eff }} \leq 150 \mathrm{~K}\right)$. Figure 3 depicts such a sequence and, in principle, spans a range in $T_{\text {eff }}$ from $\sim 2000 \mathrm{~K}$ to $\sim 100 \mathrm{~K}$. Note that near $\mathrm{T}_{\text {eff }} \sim 400-500 \mathrm{~K}$ water clouds will appear to naturally define yet another class of EGPs/brown dwarfs. 


\section{Composition Diagnostics of EGP Atmospheres}

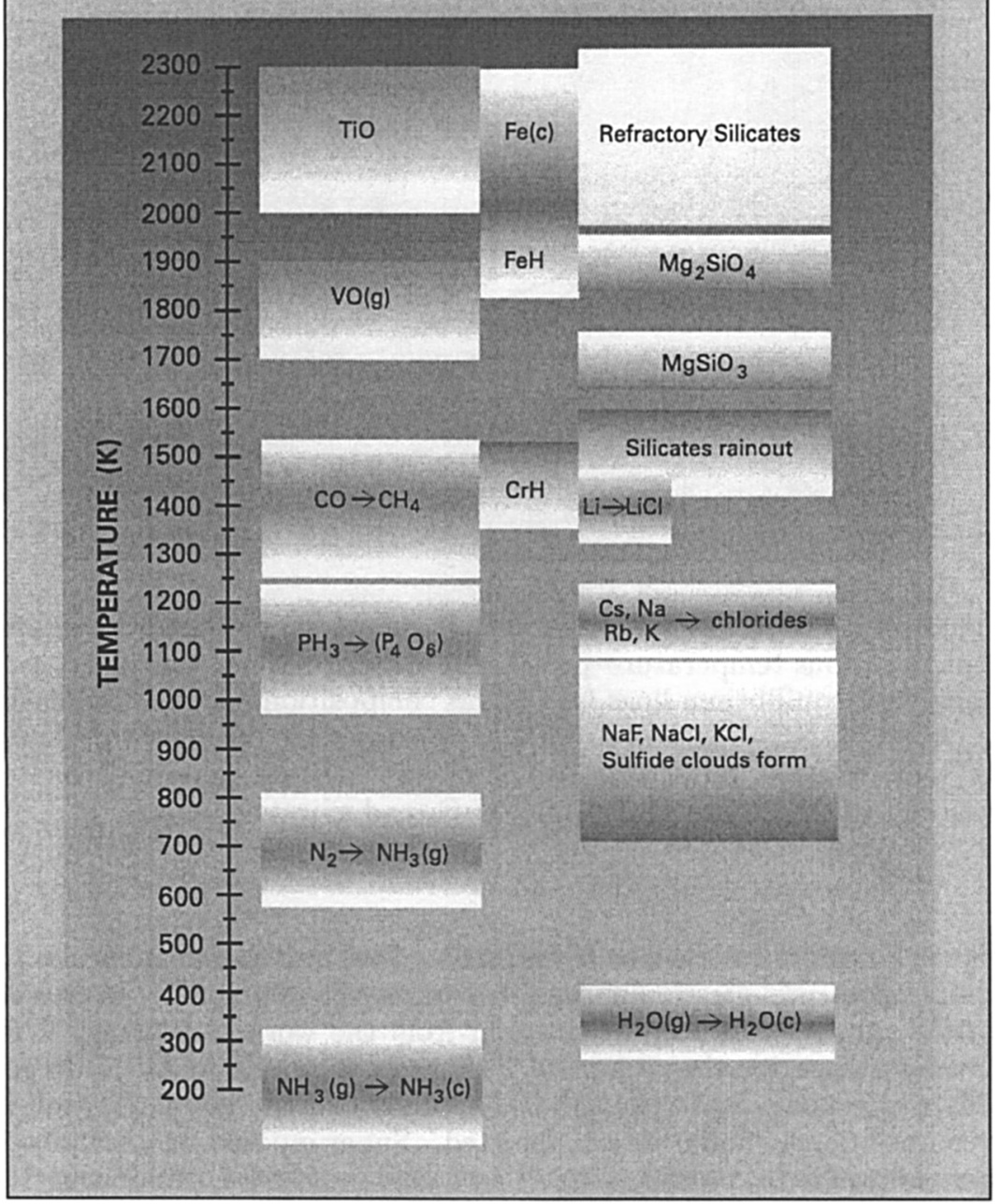

Figure 3. A crude sequence of dominant or interesting constituents of EGP and brown dwarf atmospheres, as a function of temperature. The ordering should inform the use of specific spectroscopic diagnostics for spectral typing. The species at the top are expected at higher temperatures, while those at the bottom should be found only at the lower temperatures. The approximate positions of some important compositional transitions are shown. Note the large number of interesting transitions/disappearances/appearances that could in principle be used to type SMOs from a $T_{\text {eff }}$ of $2000 \mathrm{~K}$ to $100 \mathrm{~K}$. 


\section{Abundances of the Alkali Metals and Metal Hydrides, with and without Rainout}

Rainout can affect the abundances of various spectroscopically important species in the upper atmospheres of a substellar-mass object. In particular, it can enhance the abundances of the alkali metals and $\mathrm{H}_{2} \mathrm{~S}$. True equilibrium abundances without rainout will sequester a variety of elements in refractory compounds, so that they are not as available at altitude in an SMO atmosphere. However, if silicon and aluminum, for example, are depleted from the atmosphere by the formation and settling of refractory species, they are not available at the lower temperature/pressure levels to combine with the alkali metals. The latter would otherwise form silicates (e.g. high albite and sanadine), but, being less refractory, would form them at lower temperatures than the $\sim 1500-2500 \mathrm{~K}$ range of the classical refractories. Hence, due to the rainout of silicates at depth and the related sequestration of refractory species, the abundances of the alkali metal atoms can be one to three orders of magnitude higher at temperatures less than $1200 \mathrm{~K}$ than they would have been had rainout not obtained (Lodders 1999). This may have important consequences for the spectra of SMOs (Burrows, Marley, \& Sharp 1999). The rainout of condensed Fe at temperatures near $\sim 2300$ $\mathrm{K}$ allows $\mathrm{H}_{2} \mathrm{~S}$, not $\mathrm{FeS}$, to be the dominate sulfur-bearing molecule below $\sim 750$ $\mathrm{K}$. Hence, the strengths of various lines and bands may be directly tied to the process of rainout. For instance, at $1050 \mathrm{~K}$ rainout has led to a $\sim 100$-fold enhancement in the abundance of nascent sodium and to a $\sim 10$-fold enhancement in the abundance of nascent potassium. Hence, the consequences of rainout are striking and testable.

\section{The Suppression of Flux in the Optical Spectra of Methane Dwarfs}

Marley et al. (1996) and Allard et al. (1996) concluded that Gliese 229B's spectrum longward of $\sim 1 \mu \mathrm{m}$ can be fit by a simple mixture of $\mathrm{H}_{2} \mathrm{O}, \mathrm{CH}_{4}$, and $\mathrm{H}_{2}$. However, neither Marley et al. (1996) nor Allard et al. (1996) were able to fit the near-infrared observations between $0.8 \mu \mathrm{m}$ and $1.0 \mu \mathrm{m}$ and the theoretical excesses in flux ranged from 10 to 100 . Attempts to fit this problematic spectral interval were made by Golimowski et al. (1998), who invoked a Tsuji et al. (1996) spectrum that retained $\mathrm{TiO}$ in the atmosphere to take full advantage of TiO's strong absorption in the blue, and Griffith et al. (1998), who hypothesized that a population of small photochemical haze particles analogous to the red Titan Tholins (Khare \& Sagan 1984) resided between $\sim 1400 \mathrm{~K}$ and $\sim 1800 \mathrm{~K}$ in Gl 229B's atmosphere. Indeed, $\mathrm{TiO}$ does give roughly the correct continuum slope, but it imposes the characteristic $\mathrm{TiO}$ bands on the spectrum that are not seen in either late $\mathrm{L}$ or $\mathrm{T}$ dwarfs. Furthermore, as stated above, chemical abundance studies show that $\mathrm{Ti}$ and $\mathrm{V}$ are depleted from the atmosphere to depths below the silicate clouds and to temperatures near $2000 \mathrm{~K}$, far below Gl 229B's visible atmosphere. The dependence on wavelength between $0.85 \mu \mathrm{m}$ and 0.93 $\mu \mathrm{m}$ of the imaginary index of refraction of the hazes of Griffith et al. to explain the suppression of the optical flux in Gliese $229 \mathrm{~B}$ is $\sim 5 \times$ steeper than that of known, suggested materials. 


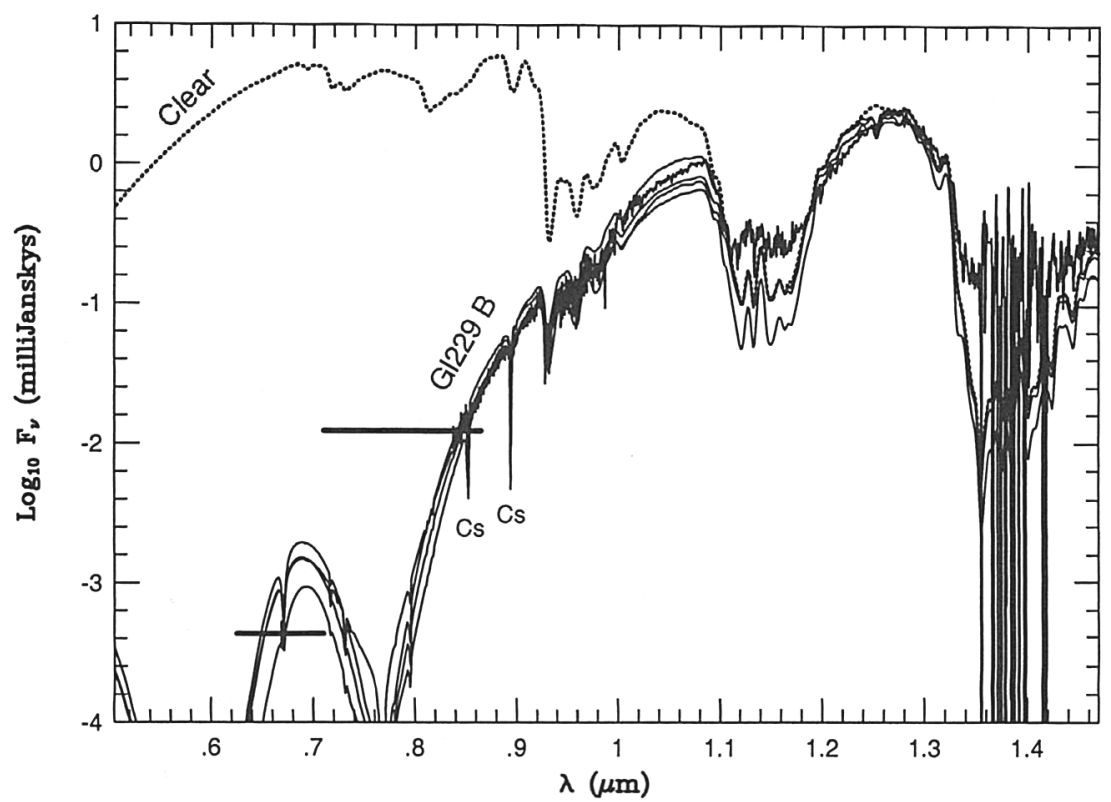

Figure 4. The $\log _{10}$ of the absolute flux $F_{\nu}$ in milliJanskys versus wavelength $\lambda$ in microns from $0.5 \mu \mathrm{m}$ to $1.45 \mu \mathrm{m}$ for Gliese $229 \mathrm{~B}$, according to Leggett et al. (1999) (heavy solid), and for four theoretical models (light solid), taken from Burrows, Marley, \& Sharp (1999). Also included is a model, denoted "Clear" (dotted), without alkali metals and without any ad hoc absorber due to grains or haze. The horizontal bars near $0.7 \mu \mathrm{m}$ and $0.8 \mu \mathrm{m}$ denote the WFPC2 $R$ and $I$ band measurements of Golimowski et al. (1998).

However, there is another, ready-made, source of continuum opacity, the red wings of the KI resonance lines at $7665 \AA$ and $7699 \AA$, that are in just the right place, shortward of the Gl 229B data, with what appear to be just the right strength and opacity slope, to fully explain the anomalous data (Tsuji et al. 1999). The KI resonance doublet comes into its own only if other sources of opacity that dominate in M dwarfs, such as $\mathrm{TiO}$ and VO, are absent. The formation of refractories and their subsequent rainout accomplishes just that. Figure 4 depicts synthetic spectra for four different models of Gl 229B that include the lines of the alkali metal atoms, in particular the K I doublet at 7700 $\AA$, employing the formalism of Burrows, Marley, \& Sharp (1999). Included is a calculation of the "Clear" atmosphere fluxes from $0.5 \mu \mathrm{m}$ to $\sim 1.45 \mu \mathrm{m}$ and the Gl 229B data are taken from Leggett et al. (1999). As Figure 4 demonstrates, the fit between $0.85 \mu \mathrm{m}$ and $1.0 \mu \mathrm{m}$ is quite good and does not seem to require a layer of red particulates.

\section{Conclusions}

The results from the solar system with its very-low temperatures and fully metaldepleted surfaces may not always carry well into the broad expanse that encom- 
passes the parameter space with which we are now confronted in studying SMOs. In addition, the truisms and formalism of classical stellar evolution, developed in the context of higher temperatures and of atoms and ions, are not ideally suited to SMO investigations. It is only by the fruitful merger of the perspectives of both planetary science and astronomy that the emerging science of EGP/BDs, spanning as it does more than two orders of magnitude in mass, a factor of 20 in effective temperature, and a factor of 1000 in age, will make real and sustained progress. These composition and abundance studies are meant to facilitate that progress by identifying the unique and diagnostic composition patterns in the atmospheres of these exotic beasts.

Acknowledgments. The author would like to thank Mark Marley, Richard Freedman, Jonathan Lunine, Bill Hubbard, David Sudarsky, Sandy Leggett, Christopher Sharp, and Jim Liebert for many useful inputs and suggestions. This work was supported in part by NASA grants NAG5-7499 and NAG5-7073.

\section{References}

Allard, F., Hauschildt, P.H., Baraffe, I., \& Chabrier, G. 1996, ApJ, 465, L123

Anders, E. \& Grevesse, N. 1989, Geochim. Cosmochim. Acta, 53, 197

Burrows, A., Hubbard, W.B., Saumon, D., \& Lunine, J.I. 1993, ApJ, 406, 158

Burrows, A., Marley M., Hubbard, W.B. Lunine, J.I., Guillot, T., Saumon, D. Freedman, R., Sudarsky, D., \& Sharp, C.M. 1997, ApJ, 491, 856

Burrows, A. \& Sharp, C.M. 1999, ApJ, 512, 843

Burrows, A., Marley, M.S., \& Sharp, C. 1999, accepted to ApJ

Fegley, B. \& Lodders, K. 1996, ApJ, 472, L37

Geballe, T.R., Kulkarni, S.R., Woodward, C.E., \& Sloan, G.C. 1996, ApJ, 467, L101

Golimowski, D.A., et al. 1998, AJ, 115, 2579

Griffith, C.A., Yelle, R.V., \& Marley, M.S. 1998, Science, 282, 2063

Khare, B.N. \& Sagan, C. 1984, Icarus, 60, 127

Kirkpatrick, J.D., Reid, I.N., Liebert, J., Cutri, R.M., Nelson, B., Beichman, C.A., Dahn, C.C., Monet, D.G., Gizis, J., \& Skrutskie, M.F. 1999, ApJ, 519, 802

Leggett, S., Toomey, D.W., Geballe, T., \& Brown, R.H. 1999, ApJ, 517, L139

Lodders, K. 1999, ApJ, 519, 793

Marley, M.S., Saumon, D., Guillot, T., Freedman, R.S., Hubbard, W.B., Burrows, A., \& Lunine, J.I. 1996, Science, 272, 1919

Nakajima, T., Oppenheimer, B.R., Kulkarni, S.R., Golimowski, D.A., Matthews, K. \& Durrance, S.T. 1995, Nature, 378, 463

Oppenheimer, B.R., Kulkarni, S.R., Matthews, K., \& Nakajima, T. 1995, Science, 270,1478

Oppenheimer, B.R., Kulkarni, S.R., Matthews, K., \& van Kerkwijk, M.H. 1998, ApJ, 502, 932

Tsuji, T., Ohnaka, K., Aoki, W., \& Nakajima, T. 1996, A\&A, 308, L29

Tsuji, T., Ohnaka, K., \& Aoki, W. 1999, ApJ, 520, L119 


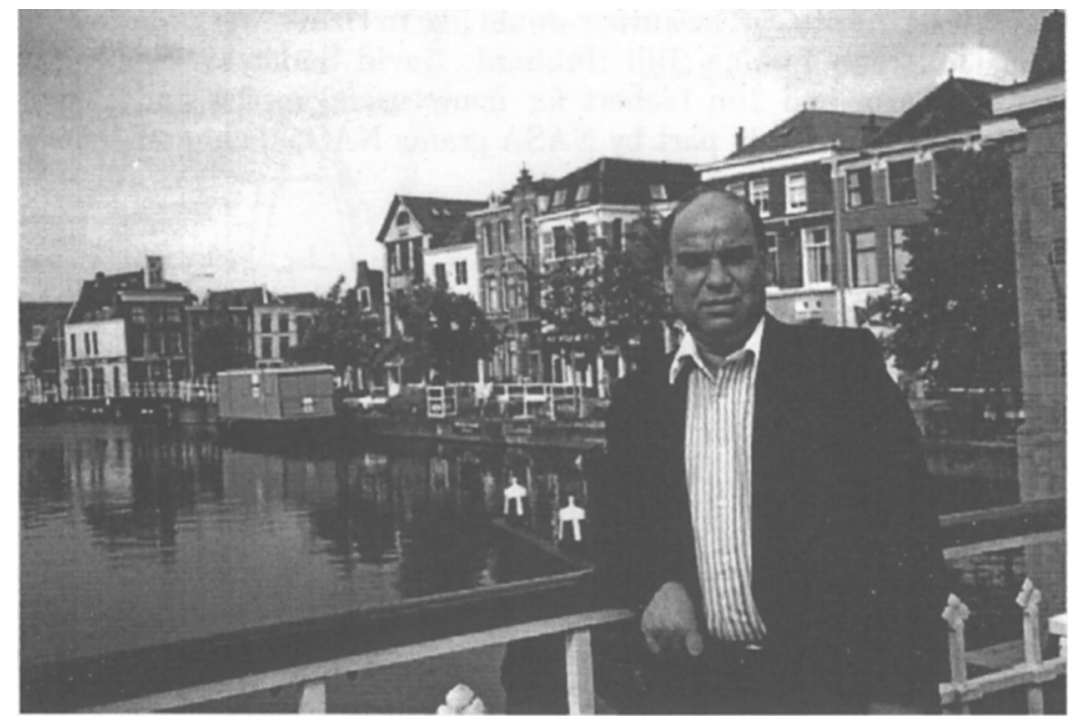

Prof. Dr. Patan Deen Singh, former member of the IAU Working Group on Astrochemistry, photographed during IAU Symposium 178 in Leiden in 1996. The Astrochemistry community is grateful for his efforts in the working group, in particular the organization of the second Symposium in this series, IAU Symposium 150 in Brazil in 1991. 